\title{
Ultra-structural Study by Transmission Electron Microscopy: Effect of Omega-3 on Ovary Cell Organelles after Experimental Induced Poly Cystic Ovary Syndrome
}

\author{
Elahe Ouladsahebmadarek ${ }^{1}$, Arash Khaki ${ }^{2 *}$
}

Article History:
Received 1 October 2013
Accepted 3 February 2014
Revised 15 January 2014
Available online 4 February 2014
Keywords:
Estradiol-Valerate
Omega-3
Ovary
PCO
Corresponding Author:
Arash Khaki, Department of
Pathology, Tabriz Branch, Islamic
Azad University, Tabriz, Iran.
Tel: +989143138399
Email: arashkhaki@yahoo.com

Article History:

Received 1 October 2013

Accepted 3 February 2014

Revised 15 January 2014

Keywords:

Omega-3

Ovary

Corresponding Author:

Arash Khaki, Department of Pathology, Tabriz Branch, Islamic Tel: +989143138399

mail: arashkhaki@yahoo.com

\begin{abstract}
Objectives: Polycystic ovary syndrome (PCOS) is the most frequent cause of female infertility, affecting about $4 \%$ to $8 \%$ of women in the age of procreation. For evaluation the protective effects of omega-3 polyunsaturated fatty acid on ovarian structure in experimental PCO induced by estradiol-valerat, this research was done.

Materials and Methods: Wistar female rats $(n=40)$ were allocated into four groups, one control $(\mathrm{n}=10)$ and three test groups $(n=30)$, that one group received omega-3 (60 $\mathrm{mg} / \mathrm{rat} / \mathrm{orally} /$ daily), second and third groups were induced PCO by single injection of estradiol-valerate $(16 \mathrm{mg} / \mathrm{kg} / \mathrm{i} . \mathrm{m})$, third group also received omega-3 $(240 \mathrm{mg} / \mathrm{kg})$ for 60 consequence days. Animals were kept in standard conditions. On day 60, the ovarian tissue of Rats in whole groups were removed and prepared for pathological analysis.

Results: vacuolated area and rough endoplasmic reticulum expanded, de-granulated, disorganized were seen in PCO groups; however, these side effects decreased in the groups that received omega-3 significantly $(\mathrm{p}<0.05)$ in comparison to experiment groups and ovarian weights in PCO experimental decreased significantly $(\mathrm{p}<0.05)$.

Conclusion: Results revealed that administration of omega-3 could significantly treat PCO. This suggested that polyunsaturated fatty acid could diminish negative side effects of PCO on ovary tissue.
\end{abstract}

1 - Women's Reproductive Health Research Center, Tabriz University of Medical Sciences, Tabriz, Iran.

2- Department of Pathology, Tabriz Branch, Islamic Azad University, Tabriz, Iran. 


\section{Introduction:}

Poly Cystic ovary syndrome (PCOS) is a common endocrine disorder in women during reproductive age (1).The prevalence of PCOS is estimated about $4 \%$ to $8 \%$ among reproductive-age women. PCOS manifestations include metabolic, reproductive, and psychological disorders. Thus, PCOS usually is along with many manifestations, including insulin resistance , hyperglycemia, hyperinsulinemia, type 2 diabetes mellitus, cardiovascular disease, hyperandrogenism, ovulatory dysfunction, infertility, increased anxiety, and depression. The phenotype varies depending on life stage of disease, genotype, ethnicity and environmental factors such as life style. The aetiology of PCOS is multifactorial probably. Diagnostic criteria rely on PCOS clinical and biochemical findings. Oxidative stress (OS) may play a role in the pathophysiology of PCOS $(1,2,3)$ Normal hormonal levels in PCOS women converts to abnormal levels; the GnRH level changes indicate in most women with PCOS, LH levels are elevated and the LH/FSH ratio is high that can be considered as a diagnostic tool for this disorder (4). Evaluation of corticosteroid hormones demonstrates estrogen level increased in PCOs women while progesterone level decreased in these women (5).

Reactive oxygen species (ROS) are formed in the human body due to metabolism and cellular function; naturally, protective mechanism of the body against ROS is antioxidants. An antioxidant is a substance that can prevent or delay OS by scavenging biological important reactive oxygen species (O2-, H2O2, OH, HOCl, ferryl, peroxyl, and alkoxyl) (6). Oxidative stress as a pathological state can be generated when there is an imbalance between pro-oxidant molecules (reactive oxygen and nitrogen species) and antioxidants defensive system. The role of $O S$ in the pathogenesis of subfertility in both male and female has been shown by some studies; moreover, environmental pollutants cause $O S$, so the role of life style is prominent in generating oxidative stress. The impact of OS on oocytes and reproductive functions remains unclear.
OS may result in pathological diseases in female reproductive tract such as poly cystic ovary syndrome; hence, the antioxidants effect is very important to decrease infertility induced by oxidative stress (7). Omega-3, $\alpha$-Linolenic acid is a poly unsaturated essential fatty acid obtained by nutrients (8). In the recent studies, the effects of $n-3$ fatty acids on the major risk factors of metabolic syndrome, especially adiposity, dyslipidemia, insulin resistance, diabetes, hypertension, oxidative stress, and inflammation have been assessed which show the beneficial effects of omega- 3 on these parameters (9). The main purpose of this study is to assess the anti-oxidative and protective effects of poly unsaturated fatty acid (PUFA) on the ROS generated in PCOS induced rats.

\section{Material \& Methods:}

\section{Animals}

Forty adult 8 week-old Wistar albino female rats weighing $250 \pm 10$ grams were obtained from Animal Facility of Pasture Institute of Iran. Rats were kept in temperature controlled rooms $(25 \mathrm{C})$ with constant humidity (40-70\%) and 12h/12h light/ dark cycle prior to use in experimental protocols. All animals were treated in accordance to the Principles of Laboratory Animal Care [NIH]. The experimental protocol was approved by the Animal Ethical Committee in accordance with the guide for the care and use of laboratory animals prepared by Tabriz medical University. All rats were fed a standard diet and water. The daily intake of animal water was monitored at least one week prior to start of treatments in order to determine the amount of water needed for each experimental animal. Thereafter, the rats were randomly selected and divided into control $(n=10)$ and test groups $(n=30)$, that subdivided into groups of 3 , one group received omega-3 (240 $\mathrm{mg} / \mathrm{kg} /$ orally/daily), second and third groups were induced PCO by single injection of estradiol-valerate $(16 \mathrm{mg} / \mathrm{kg} / \mathrm{i} . \mathrm{m})$, third group of this research received omega- 3 ( $240 \mathrm{mg} / \mathrm{kg}$ ), for 60 days. Animals were kept in standard conditions. On day 60, the ovarian tissues of Rats in whole groups were removed for pathology analysis. 


\section{PCO induction}

Thirty days before the experimental procedure, twenty rats were each given a single intra muscular (i.m.) injection of $4 \mathrm{mg}$ EV (Riedeldehaen, Germany) solved in 0.2 $\mathrm{ml}$ oil in order to induce PCO (PCO group).

Surgical Procedure

On day 60, (at the end of the treatment period), the blood samples in control \& experimental groups were immediately obtained.

\section{Histopathology of Ovary}

The ovarian tissues were fixed in $10 \%$ buffer formalin and embedded in paraffin wax. Five micron thick sections were obtained and prepared and then stained with masson's trichrome stain (T.C) .The specimens were examined using an Olympus $3 \mathrm{H}$ light microscope.

\section{Transmission electron microscopy}

For transmission electron microscopy (TEM), the ovary samples were cut into pieces $(2 \times 2 \mathrm{~mm})$ and fixed in $2.5 \%$ glutaraldehyde $(\mathrm{PH}=7.4)$ for $6-8 \mathrm{hr}$ at $4^{\circ} \mathrm{C}$. They were washed and post fixed in $2 \%$ OSO 4 for $1 \mathrm{hr}$, at $4^{\circ} \mathrm{C}$. The tissue was dehydrated through ascending grades of ethanol and embedded in araldite CY212. Semi thin sections $(1 \mu \mathrm{m})$ were cut and stained with toluidine blue. Ultra-thin sections $(60-70 \mathrm{~nm})$ were cut and stained with uranyl acetate and alkaline lead citrate.

\section{Statistical analysis}

Statistical analysis was done using the ANOVA test for comparison of data in the control group with the experimental groups. The results were expressed as mean \pm S.E.M (standard error of means). P-values less than 0.05 were considered significant and are written in the parentheses.

\section{Results:}

In the ovaries of the control group, thickness of zona pellucida and distribution of microvilli as well as the other organelles like mitochondria and rough endoplasmic reticulum were normal. Intercellular junction complex between corona radiata cells was intact. The nuclei of granulosa cells showed euchromatin appearance and prominent nucleolus. Normal mitochondria, rough endoplasmic reticulum and lipid droplet was obviously seen within the cytoplasm of granulosa cells. Following 60 days after inducing PCO by single injection of estradiol-valerate (16mg/ kg/ i.m), thickness of zona pellucida and distribution of microvilli was negatively affected and vacuolated area, rough endoplasmic reticulum was expanded, degranulated, disorganized, and even showed signs of rupture but intercellular junction complex was still observable. In PCO groups receiving omega-3, Nuclei of luteal cells were euchromatin with obvious nucleolus and still mitochondria were normal. Nuclear envelop dilation was the common finding of PCO damages which was seen after 60 days. Photomicrographs of ovary tissue in control group show normal structure of follicles, masson's trichrome staining, X160 , ovary tissue in of $\omega$-3 group show normal structure of follicle, masson's trichrome staining, X160. Other slide showed ovary tissue of PCO group cyst (arrow) and hyperemia(star), masson's trichrome staining, X160,figures:1,2,3.

\section{Discussion:}

Reactive oxygen species (ROS) and antioxidants are in balance in a healthy body. Reactive oxygen species is a phrase used to describe a variety of molecules and free radicals (chemical species with one unpaired electron) derived from molecular oxygen $(10,11)$. In the reproductive tissue, the elevated level of ROS generated by active metabolism and steroidogenesis may cause oocyte and DNA damage; besides, the ROS play a physiological role during ovulation that is similar to some responses to inflammation. ROS may play a role in the regulation of growth of ovarian mesenchyme in a pathological condition such as PCOs that excessive oxidative stress may contribute to ovarian mesenchyme hyperplasia. One of the protective mechanisms of the body against the ROS effect is antioxidant enzymes such as superoxide dismutase (SOD) and glutathione peroxidase (GPX) for eliminating the ROS. ROS can activate scavenging system and Redox system that could repair oxidized and damaged molecules by using NADPH as an original electron source $(11,12)$. The ROS cause lipid peroxidation, so it can damage DNA and/or change cell signalling and cellular function. 
The oxidative stress (OS) leads to DNA damage of ovarian epithelium or cell apoptosis; However, oxidative status of the cell modulates follicular growth, corpus luteum formation, endometrial differentiation and embryonic growth. Oxidative stress may also be the cause of preeclampcia, abortion, endometriosis, PCOS, infertility, mole hidatyform, radicalinduced birth defects; hence, evaluating protection against OS is very important in reproductive science.

Recent findings illustrated beneficial influence of oxygen radicals and ROS in many physiological states like development of germ cells in the uterine environment, oocyte maturation, ovulation, and corpus luteum function and regression .Oxidative stress (OS) may play a role in the pathophysiology of PCOS. OS may affect insulin resistance (IR) that is common in young non-obese PCOS women $(11,12,13)$. ROS generation from mononuclear cells (MNCs) in response to hyperglycemia increased in PCOS independent of obesity. The resultant oxidative stress may contribute to a pro-inflammatory state that induces insulin resistance and hyperandrogenism in women with this disorder (14). Antioxidants as defensive mechanism of the body modulate the reactive oxygen species effect, and they also prevent oxidative stress. Enzymatic antioxidant defences include superoxide dismutase (SOD), glutathione peroxidase (GPx), catalase (CAT). Non-enzymatic antioxidants include ascorbic acid (Vitamin C), $\alpha$-tocopherol (Vitamin E), glutathione (GSH), carotenoids, flavonoids, and other antioxidants.

There is a balance between both the activities and the intracellular levels of these antioxidants normally that is essential for the organisms health (15) .In this study on rats we subdivided female rats to one control group and three experimental groups , first group just received omega-3 , PCOS was induced by estradiol-valerate in second group (16 mg/ kg/ i.m) and third group was injected estradiol-valerate and omega-3; the experiment duration was 60 days. After 60 days pathological analysis showed decreasing in thickness of zona pellucida and distribution of microvilli in the group injected estradiol valerate ; in addition, in this group, expansion, degranulation and disorganization of vacuolated area and rough endoplasmic reticulum were obvious while in third group these findings decreased, mitochondria was normal and nuclei appearance of granulosa cells was euchromatin with prominent nucleolus .

This study could be used as an evidence for confirmation of recent studies about the omega-3 effect on ovary tissue (16). Superoxide dismutase (SOD) produced in cumulus oophorus cells is closely associated with oocyte maturation and also GPx and catalase are significant for reproduction health; moreover, GSH as a redox system exists in a large amount in the oocytes, so according to this study, it seems that omega3 may modulate the ROS effect by affecting on these anti-oxidative systems but it is necessary more studies to confirm this idea $(12,17,18)$. Superoxide (02 -), hydrogen preoxide (H202), and lipid peroxides are generated in luteal tissue during natural and prostaglandin-induced regression in the rat. The cellular origin of oxygen radicals in regressing corpora luteal is predominately from resident and infiltrated leukocytes, notably neutrophils. Despite physiological roles of oxidative stress, the higher production of these agents may lead to an increased risk of ovarian pathology and it would probably be exacerbated under conditions of reduced antioxidant status (19). Some studies indicated that oxygen radicals may function as intracellular regulators of steroidogenesis in the corpus luteum (20).Oxidative stress and depletion of the antioxidant glutathione (GSH) cause apoptosis in many systems. Previous works showed that antioxidants prevented apoptosis effectively (21). Apoptosis, a type of physiological or active cell death, has been implicated as a mechanism underlying regression of the corpus luteum (CL) in the rat, bovine, rabbit and ovine ovary. Reactive oxygen species play an important role in luteolysis in rodent ovaries (22). The decreased levels of Paraoxonase lactonase 
(antioxidant enzyme), platelet-activating factor acetylhydrolase (antioxidant enzyme preventing LDL oxidation), but elevated asymmetric dimethylation (ADMA as an inhibitor of nitric oxid synthesis) might be a marker of development of atherosclerosis in the PCOS patients associated with insulin resistance (23). Paraoxonase 1 (PON1) as an enzymatic antioxidant prevents lipid peroxidation. According to some researches, there is a direct relation between increased malondialdehyde (MDA) and decreased antioxidant (PON1) activity and total antioxidant capacity (TAC). Since PON1 activity is lower in the PCOS women, dyslipidemia is common in these women; it may cause lower-density-lipoprotein ( LDL) oxidation in arterial walls, so atherogenesis is also common in the PCOS women $(24,25)$. Many studies about the effects of vitamin D and its derivatives on the hyperandrogenism, menstrual irregularity, infertility, insulin resistance (IR), and oxidative stress showed the therapeutic and protective effect of vitamin D3,25 hydroxy vitamin $\mathrm{D}$, and also calcium in the PCOS women (26-28).

The protective effect of vitamin $\mathrm{E}$ against oxidative stress has been shown in the improvement of cardiovascular diseases (29). $\alpha$-Linolenic acid is an organic compound (carboxylic acid) found in many vegetable oils and fish oil; The other name of $\alpha$-Linolenic acid is -cis-9,12,15octadecatrienoic acid 18:3 (n-3). (three cis double bond) (30). Polyunsaturated fatty acids omega-3 (PUFA), particular eicosapentaenoic (EPA) and docosahexaenoic acid (DHA), are bioactive lipids that positively impact on signalling pathways involved in the development of cardiovascular diseases, so cardioprotective action of PUFA omega- 3 has been approved $(31,32)$. Omega-3 may increase high density lipoprotein cholesterol (HDL), but it may decrease the triglyceride levels. In the women with PCOS, omega-3 can modify the adiponectin (a soluble matrix protein) level, insulin resistance, and lipid profiles $(33,34,35)$.

\section{Conclusion :}

Supplementation with omega-3 fatty acids has beneficial effects on serum lipid peroxidation and antioxidant enzymes in addition which may lead to decreased rate of occurrence of vascular complications in diabetes patients. The anti-inflammatory effect of $n-3$ fatty acids may also supress the oxidative stress. Supplementation with antioxidants has been proposed to counteract oxidative stress and improve antioxidant status of the insulin resistance common in the PCOS women. In many studies, increased dietary PUFA intake was significantly associated with beneficial metabolic and endocrine effects in women with PCOS .

\section{Conflicts of interest:}

Authors declare that there is no any conflict of interest.

\section{Acknowledgments:}

We would like to thank authorities of Women's Reproductive Health Research Center, Tabriz University of Medical Sciences for the scientific and ethical approval and financial support of this research. 


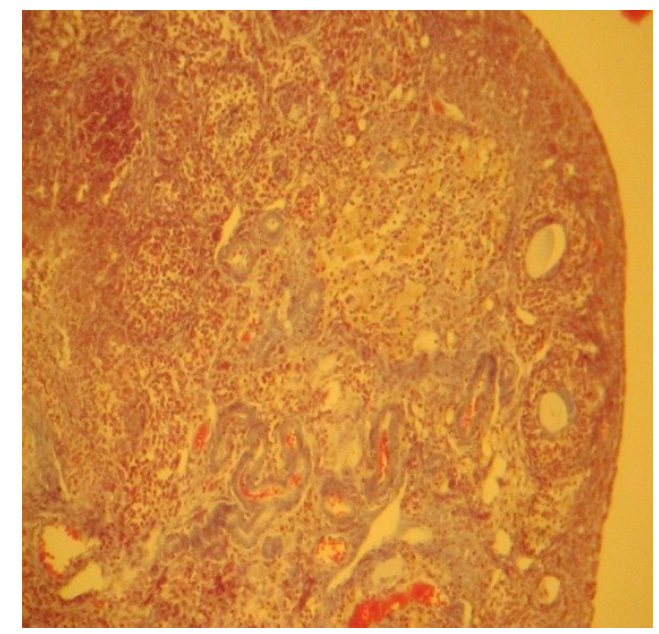

Figure 1. Photomicrograph of ovary tissue of control group shows normal structure of follicle, T.C staining, X160.

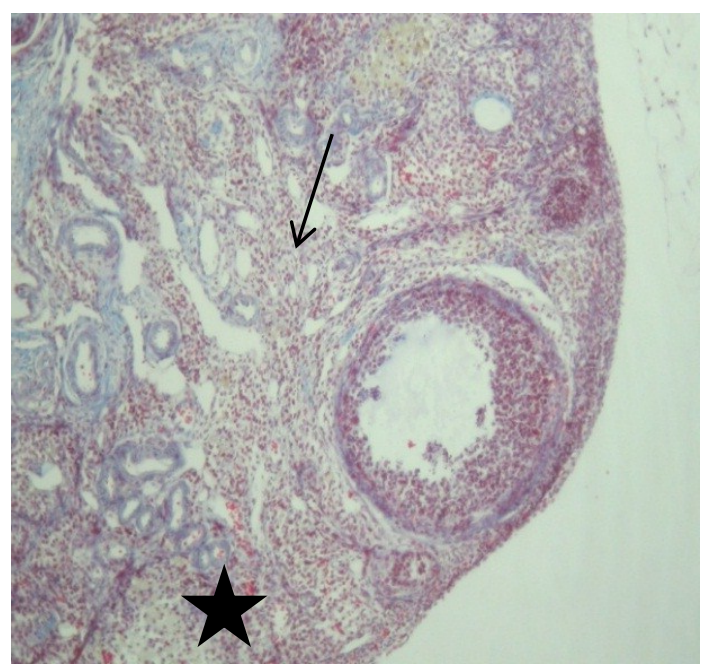

Figure 3. Photomicrograph of ovary tissue of PCO group shows cyst (arrow) and hyperemia (star),T.C staining, X160.

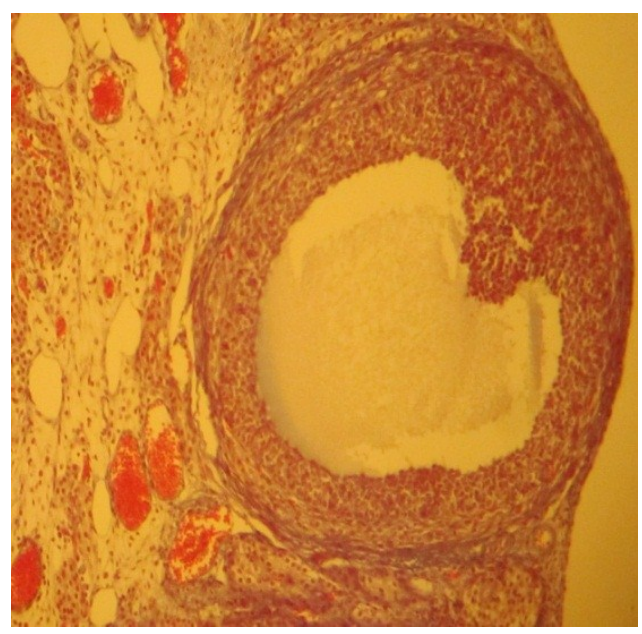

Figure 2 Photomicrograph of ovary tissue of $\omega$-3group shows normal structure of follicle, T.C staining, $\mathrm{X} 160$.

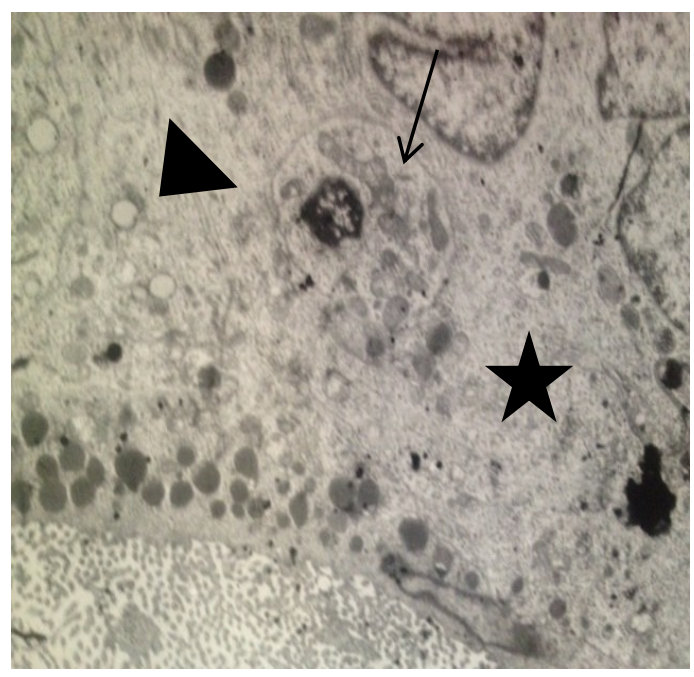

Figure 4. Electron micrograph of ovary tissue of PCO group shows cyst (triangle) and dilated mitochondria (arrow) and fibrosis area (Star),X4000. 
Table 1. Effect of Omega-3, fatty acids on ovarian tissue Fibrosis, cyst, mitochondrial dilatation and ovary weight of control in poly cystic ovarian (PCO) rats.

\begin{tabular}{|c|c|c|c|c|}
\hline Groups & Control & $\begin{array}{c}\text { Omega-3, fatty } \\
\text { acids }\end{array}$ & PCO & $\begin{array}{c}\text { PCO plus,1cc } \\
\text { Omega-3, fatty } \\
\text { acids /rat }\end{array}$ \\
\hline ovary (gr) & $\begin{array}{c}1.09 \pm \\
0.55\end{array}$ & $1.88 \pm 0.54$ & $0.07 \pm 0.55^{*}$ & $0.09 \pm 0.55$ \\
\hline Fibrosis (\%) & $0.00 \pm 0.55$ & $0.00 \pm 0.01$ & $3.11 \pm 0.55^{*}$ & $2.10 \pm 0.56^{*}$ \\
\hline $\begin{array}{c}\text { Mitochondrial } \\
\text { dilation (\%) }\end{array}$ & $0.00 \pm 0.05$ & $0.01 \pm 0.10$ & $6.70 \pm 0.55^{*}$ & $3.3 \pm 0.55^{*}$ \\
\hline Percent of cysts (\%) & $0.02 \pm 0.11$ & $0.01 \pm 0.11$ & $10.87 \pm 0.11^{*}$ & $5.01 \pm 0.11^{*}$ \\
\hline
\end{tabular}

Data are presented as mean \pm SE.

*Significant different at $\mathrm{P}<0.05$ level, (compared with the control group).

\section{References:}

1. Franks S. Polycystic Ovary Syndrome. J Med. 1995; 333 (13): 853-861.

2. Huang A, Brennan K, Azziz R. Prevalence of Hyperandrogenemia in the Polycystic Ovary Syndrome Diagnosed by the NIH 1990 Criteria .J Fertil Steril. 2010; 93(6): 1938-1941.

3. Teede H, Deeks A, Moran L. Polycystic ovary syndrome: a complex condition with psychological, reproductive and metabolic manifestations that impacts on health across the lifespan . J freeradbiomed. 2007; 43(10):1388-1393.

4. Lewandowski KC, Cajdler-Łuba A, Salata I, Bieńkiewicz M, Lewiński A .The utility of the gonadotrophin releasing hormone $(\mathrm{GnRH})$ test in the diagnosis of polycystic ovary syndrome (PCOS). J endokrynol pol. 2011; 62(2): 120-128.

5. Carmen L. Pastor, Marie L. Griffin-Korf, Joseph A. Aloi, William S. Evans and John C. Marshall .Polycystic Ovary Syndrome: Evidence for Reduced Sensitivity of the GonadotropinReleasing Hormone Pulse Generator to Inhibition by Estradiol and Progesterone. J Clin Endocrinol Metab. 1998; 83(2): 582-590.

6. Halliwell B. Reactive oxygen species in living systems: source, biochemistry, and role in human disease. Am J Med. 1991; 91(3): S14-S22.

7. Agarwal A, Aponte-Mellado A, Premkumar B J, Shaman A, Gupta, S. The effects of oxidative stress on female reproduction: a review. Reprod biol endocrino. 2012; 10(1): 49-80

8. Wathes DC, Abayasekara DRE, Aitken RJ. polyunsaturated Fatty Acids in Male and Female Reproduction . Biol Reprod. 2007; 77(2): 190-201.

9. Poudyal H, Panchal SK, Diwan V, Brown L. Omega-3 fatty acids and metabolic syndrome: effects and emerging mechanisms of action. Prog Lipid Res. 2011; 50(4): 372-387. 
10. Turrens JF. Mitochondrial formation of reactive oxygen species. J physiol. 2003; 552(2): $335-344$.

11. Agarwal A, Gupta S, Sharma R K. Role of oxidative stress in female reproduction. J Reprod Biol Endocrinol. 2005; 3(28): 1-21.

12. Fujii J, Iuchi Y, Okada F. Fundamental roles of reactive oxygen species and protective mechanisms in the female reproductive system. Reprod biol endocrinol. 2005; 3(1): 43-52.

13. Riley JC, Behrman, HR. Oxygen radicals and reactive oxygen species in reproduction. J Exp Biol Med. 1991;198 (3): 781-791.

14. González F, Rote NS , Minium J , Kirwan JP. Reactive oxygen species-induced oxidative stress in the development of insulin resistance and hyperandrogenism in polycystic ovary syndrome. J Clin Endocrinol Metab. 2006; 91(1): 336-340.

15. Valko M, Leibfritz D, Moncol J, Cronin MT, Mazur M, Telser J. Free radicals and antioxidants in normal physiological functions and human disease.Endocrinol Invest. $\mathbf{J}$ Int $\mathbf{J}$ Biochem Cell Biol. 2007; 39(1): 44-84.

16. Ouladsahebmadarek E, Khaki A, Khanahmadi SH, Ahmadi Ashtiani H, Paknejad P, Ayubi MR. Hormonal and metabolic effects of polyunsaturated fatty acid (Omega-3) on polycystic ovary syndrome induced rats under diet. Iran J Basic Med Sci. 2014; 17:in press

17. Matos L, Stevenson D, Gomes F, Silva-Carvalho JL, Almeida H. Superoxide dismutase expression in human cumulus oophorus cells. J Mol. Hum. Reprod. 2009; 15 (7): 411-419.

18. Dincer Y, Akcay T, Erdem T, Ilker Saygili E, Gundogdu, S. DNA damage, DNA susceptibility to oxidation and glutathione level in women with polycystic ovary syndrome. Scand J Clin Lab Invest. 2005; 65(8): 721-728.

19. Behrman HR, Kodaman PH, Preston SL, Gao S. Oxidative stress and the ovary. J Soc Gynecol Investig. 2001; 8(1):40-42.

20. Carlson JC, Wu XM, Sawada M. Oxygen radicals and the control of ovarian corpus luteum function . Free Radic Biol Med. 1993;14(1): 79-84.

21. Tsai-Turton M, Luderer U. Opposing effects of glutathione depletion and folliclestimulating hormone on reactive oxygen species and apoptosis in cultured preovulatory rat follicles. J Endocrinol. 2006 ;147(3): 1224-1236.

22. Rueda BR, Tilly KI, Hansen TR, Hoyer PB, Tilly JL. Expression of superoxide dismutase, catalase and glutathione peroxidase in the bovine corpus luteum: evidence supporting a role for oxidative stress in luteolysis. J Endocrine. 1995; 3(3): 227-232.

23. Bayrak T, Dursun P, Bayrak A, Gültekin M, Kolusarı A, Cakır E, et al. lactonase activity (PON-HTLase), asymmetric dimethylarginine (ADMA) and platelet activating factoracetylhydrolase (PAF-AH) activity in non-obese women with PCOS. J Gynecol Endocrinol. 2012; 28(11): 874-878. 
24. Bayram F, Kocer D, Ozsan M, Muhtaroglu S. Evaluation of endothelial dysfunction, lipid metabolism in women with polycystic ovary syndrome: relationship of paraoxonase 1 activity, malondialdehyde levels, low-density lipoprotein subfractions, and endothelial dysfunction. J Gynecol Endocrinol. 2012; 28(7): 497-501.

25. Mohamadin AM, Habib FA, Elahi TF. Serum paraoxonase 1 activity and oxidant/antioxidant status in Saudi women with polycystic ovary syndrome. J pathophys. 2009; 17(3): 189-196.

26. Wehr E, Pieber TR, Obermayer-Pietsch B. Effect of vitamin D3 treatment on glucose metabolism and menstrual frequency in polycystic ovary syndrome women. $\mathrm{J}$ Endocrinol Invest. 2011; 34(10):757-763.

27. Sára L, Nádasy G, Antal P, Szekeres M, Monori-Kiss A, Horváth EM, et al. Arteriolar biomechanics in a rat polycystic ovary syndrome model - effects of parallel vitamin D3 treatment. J Acta Physiol Hung. 2012; 99(3): 279-288.

28. Firouzabadi Rd, Aflatoonian A, Modarresi S, Sekhavat L, MohammadTaheri S. Therapeutic effects of calcium \& vitamin D supplementation in women with PCOS. J omplement Ther Clin Pract. 2012; 18(2):85-88.

29. Roberts II LJ, Oates JA, Linton MF, Fazio S, Meador BP, Gross MD, et al. The relationship between dose of vitamin $\mathrm{E}$ and suppression of oxidative stress in humans. $\mathrm{J}$ freeradbiomed. 2007; 43(10):1388-1393.

30. Beare-Rogers J , Dieffenbacher A, Holm JV. Lexicon of lipid nutrition. J Pure Appl. Chem. 2001; 73(4): 685-744.

31. Duda MK. Polyunsaturated fatty acids omega-3 as modulators of intracellular signaling pathways. J Postepy biochemii. 2012; 58(2): 149-54.

32. Wang SP, Chen YH, Li H. Association between the levels of polyunsaturated fatty acid and blood lipids in healthy individuals. J Exp Ther Med. 2012 ;4(6): 1107-1111.

33. Kesavulu MM, Kameswararao B, Apparao Ch, Kumar EG, Harinarayan CV.Effect of omega-3 fatty acids on lipid peroxidation and antioxidant enzyme status in type 2 diabetic patients. J Diabetes Metab. 2002; 28(1): 20-26.

34. Mather KJ, Kwan F, Corenblum B. Hyperinsulinemia in polycystic ovary syndrome correlates with increased cardiovascular risk independent of obesity. J Fertil steril. 2000; 73(1): 150-156.

35. Kasim-Karakas SE, Almario RU, Gregory L, Wong R, Todd H, Lasley BL. Metabolic and endocrine effects of a polyunsaturated fatty acid-rich diet in polycystic ovary syndrome. $\mathrm{J}$ Clin Endocrinol Metab. 2004; 89(2): 615-620. 